\title{
Resarch on six-component strain balance for thrust vector measurement of the mini aero-engine
}

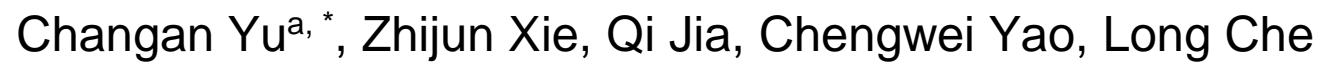 \\ Facility Design and Instrumentation Institute China Aerodynamics Research and Development \\ Center, Mianyang 621000, China. \\ achangan1234@163.com
}

\begin{abstract}
Keywords: mini aero-engine, thrust vector measurement, six-component balance, finite element analysis
\end{abstract}

\begin{abstract}
The elastomer of integral box six-component strain balance is processed by the whole piece of elastic material. The box six-component strain balance is mainly composed of a fixed frame, a floating frame, measuring elements and elastic linkage mechanisms. Seven cantilever measuring elements with rectangular cross-section are fastened on the fixed frame, and connect with the floating frame though the corresponding elastic linkage mechanisms. Thrust vector is decomposed into three directions of orthogonal force and moment. Due to such problems of mini aviation engine as relatively thrust vector, each component of force and moment mismatch, and relatively large vibration and so on, in this paper, the box six-component strain balance is firstly adopted to carry out the thrust vector measurement of the mini aero-engine. The layout of balance and the optimization design of measuring element parameters are presented considering the sensitivity and stiffness of the balance for the mini aero-engine. Finite element analysis shows that the sensitivity of the measuring element for the corresponding component is much higher than that of other components, and the interference between the measuring elements are better blocked by the elastic linkage mechanisms. The balance of stiffness and modal analysis indicates that the rigidity of the balance is relatively high without the elastic correction; and the inherent frequency is $249 \mathrm{~Hz}$ for meeting the design requirement. The calibration result of the balance shows that the measuring error of the balance for each component is lower than $0.3 \%$, and the error of the measuring accuracy is lower than $0.5 \%$. The simulated and experimental results demonstrate that the six-component strain balance meets the requirements of thrust vector measurement for the mini aero-engine.
\end{abstract}

\section{Introduction}

The mini aero-engine is characterized by the small size, simple structure, short development period low fuel consumption, and low cost etc. As military and civilian model aircraft power plant, the mini aero-engine is widely used and rapidly developed [1-2]. With the development of aero-engine thrust vector and the growth of aviation demand, the investigation and improvement of mini aero-engine is more and more important. There are mainly two types of sensors for the measurement of thrust vector of the mini aero-engine, which are piezoelectric and strain sensors. The piezoelectric sensor has the advantages of simple structure, small volume, large stiffness, high sensitivity, long longevity, fast response; however, it has low accuracy, charge leakage, low damping, zero drift and other defects [3]. The piezoelectric sensor is suitable for measuring the dynamic and impact forces, which is mainly applied in the thrust vector measurement of the rocket engine. Such as the thrust vector measuring system of the rocket engine was developed by Dalian University of Technology, and the core of the measuring components is four three-dimensional piezoelectric quartz sensors [4]. The strain senor has the advantages of good insulation performance, wet-resistance, heat resistance, high static measurement accuracy and convenient maintenance; however, the frequency response of the strain sensor is slower than that of the piezoelectric sensor. The strain sensor has been successfully applied in the thrust vector measurement of the aero-engine, Such as the six-component measuring force systems of the aero-engine vector nozzle was developed by Aerodynamics Research and 
Development Center, and the core of the measuring components is box six-component strain balance [5-6].

Although the thrust vector measurement of the mini aero-engine is dynamic measurement, the measurement time is relatively long in the experiment. Thus, the six-component strain balance is adopted to carry out the thrust vector measurement of the mini aero-engine. In this paper, the layout of balance and the optimization design of measuring element parameters are presented. The balance of stiffness and modal analysis are analyzed. The simulated and experimental results demonstrate that the six-component strain balance meets the requirements of thrust vector measurement for the mini aero-engine.

\section{Balance Structure}

The characteristics of thrust vector of the mini aero-engine are mainly reflected in the following aspects: (1) the thrust is relatively small; (2) each component is unmatched; (3) the vibration is relatively large. The balance should be reasonably designed to achieve the balance of range and rigidity according to the above characteristics of the mini aero-engine. The box six-component strain balance can relative thoroughly decomposed force and torque, and have high stiffness to resist interference factors. The strain balance is highly accurate and stable performance. Thus, the six-component strain balance is chosen to measure the thrust vector of the mini aero-engine. As far as box balance, there are two basic types: assembly balance and integral balance. The assembly balance is that the components of the balance are assembled together to form an assembling body. The assembly balance is generally applicable to measurement experiment of the large dimension and range. The integral balance is that the elastomer of the balance is processed by the whole piece of elastic material, and the dimension is relatively small. As the dimension and thrust be produced of the mini aero-engine are relatively small, thus, the unitary box balance is adopted for thrust vector measurement of the mini aero-engine. The balance for the mini aero-engine is composed of a fixed frame, a floating frame, measuring elements and elastic linkage mechanisms; and the structure schematic of the balance is shown in Fig 1. The measuring elements with rectangular cross-section are fastened on the fixed frame, and connect with the floating frame though the corresponding elastic linkage mechanisms. The thrust produced by the mini aero-engine is transmitted to the measuring elements within the elastic linkage mechanism, and then the thrust vector is decomposed into three directions of orthogonal force and moment. There are seven measuring elements in the balance for thrust measurement of the mini aero-engine. There is an axial measuring element for measuring the axial force $F_{x}$; two transverse measuring elements for measuring the lateral force $F_{z}$ and offset moment $M_{y}$; four normal measuring elements for measuring the normal force $F_{y}$, rolling moment $M_{x}$ and pitching moment $M_{z}$.

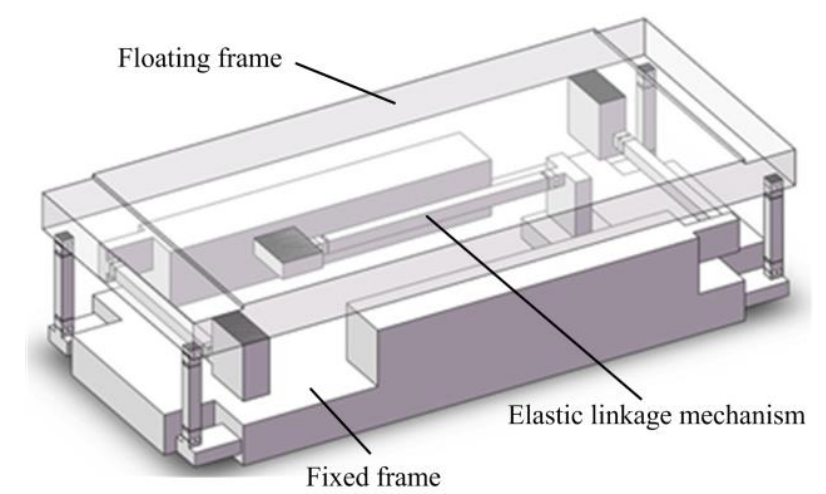

Fig. 1 Scheme of the balance for the mini aero-engine.

The elastic linkage mechanism is used to connect the floating frame and the measuring element, which transfers force and guarantees the force unipolarity (as shown in Fig. 2). The structure of dual-arc dual-direction elastic hinge is employed for the elastic linkage mechanism, which has high sensitivity, well mechanical decomposition, highly buckling critical load under the pressure, high 
process accuracy etc. Although there are some restoring force and moment, the deformation of the balance is small and the structure of the elastic linkage mechanism is always in the elastic deformation. Thus, the restoring force and moment produced by the elastic linkage mechanism have no impact to the thrust vector measurement of the mini aero-engine.

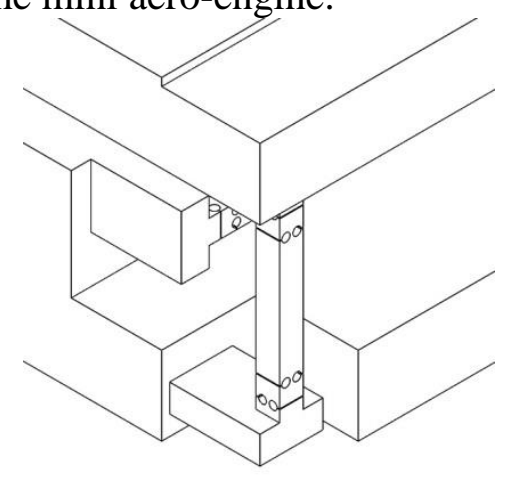

Fig. 2 Scheme of the elastic linkage mechanism of the balance.

\section{Design and Calculation For Balance Components}

\subsection{Load Calculation of The Measuring Elment}

Under the condition of ignoring the restoring force and moment caused by the deformation of the elastic linkage mechanism, the load can be decomposed by the measuring element according to the following formulas.

The load of the measuring element in the axial direction is as follows

$F_{F x}=F_{x}$

Where $F_{F x_{-}}$is the total axial force, $F_{x}$ is the metrically axial force of each measuring element.

The load of the measuring element in the transverse direction is as follows

$F_{F z}=F_{z} / 2$

$F_{M y}=M_{y} / L_{M y}$

Where $F_{F z}$ is the total lateral force, $F_{z}$ is the metrically lateral force of each measuring element, $F_{M y}$ is the total offset force, $M_{y}$ is the total offset moment, $L_{M y}$ is the distance of the normally elastic linkage mechanisms in the axial direction.

The load of the measuring element in the normal direction is as follows

$F_{F y}=F_{y} / 4$

$F_{M z}=M_{z} / 2 L_{M z}$

$F_{M x}=M_{x} / 2 L_{M x}$

Where $F_{F y}$ is the total normal force, $F_{y}$ is the metrically normal force of each measuring element, $F_{M z}$ is the total pitching force, $M_{z}$ is the total pitching moment, $L_{M z}$ is the distance of the normally elastic linkage mechanisms in the normal direction. $F_{M x}$ is the total rolling force, $M_{z}$ is the total rolling moment, $L_{M z}$ is the distance of the normally elastic linkage mechanisms in the lateral direction.

\subsection{Determination of Geometric Parameters of The Measuring Element}

The geometric parameters of the measuring element are mainly the width $b$, the thickness $h$, and the distance $l$ between the center line of the elastic linkage mechanism and the root of the cantilever beam. Based on reasonable choices of the balance range, the balance strain value, the balance structure, the strain gauge size and measuring circuit and so on, the parameters of the width $b$ and the distance $l$ can be determined. Then, the parameter of the thickness $h$ can be determined according to the following formula.

$$
\varepsilon=\frac{6 F l}{E b h^{2}}
$$

Where $\varepsilon$ is the balance strain value, $F$ is the maximum load acting on the measuring elements in one direction. 


\subsection{Strain Calculation of The Measuring Element}

In order to increase the voltage signal output, the strain gauge is attached on the root of the measuring element, and the length of sensitive gate of the strain gauge should be small as far as possible. The average strain can be obtained according to the following formula.

$$
\varepsilon_{c p}=\frac{2 l-l_{\mathrm{g}}-2 a}{2 l} \varepsilon_{\max }
$$

Where $\varepsilon_{\text {max }}$ is the maximum strain under the measuring load, $\varepsilon_{c p}$ is the average strain from the strain gauge, $l$ is the length of the measuring element, $l_{g}$ is the length of the sensitive gate, $\alpha$ is the distance between the end of strain gauge and the root of measuring element.

The measuring element of the balance is fixed on the fixed frame. The thrust produced by the mini aero-engine is transferred to the fixed frame, and the elastic linkage mechanism transfer tension and pressure. Thus, the measuring is simplified as a cantilever beam in the strain calculation. The average strain $\varepsilon_{c p}$ can be obtained according to the formulas (7) and (8).

$$
\varepsilon_{c p}=\frac{3 F\left(2 l-l_{\mathrm{g}}-2 a\right)}{E b h^{2}}
$$

\section{Finite Element Analysis}

\subsection{Strain Analysis of The Balance In Single Direction}

MSC is an advanced finite element software, which can be widely used in structure and modal analyses of products. In order to research the balance deeply, the software of MSC is chosen to conduct numerical simulations. In condition of the technical requirements and the actual working condition, the deformation of the sensor in every direction is shown in Fig. 3 under the force and moment of each component.

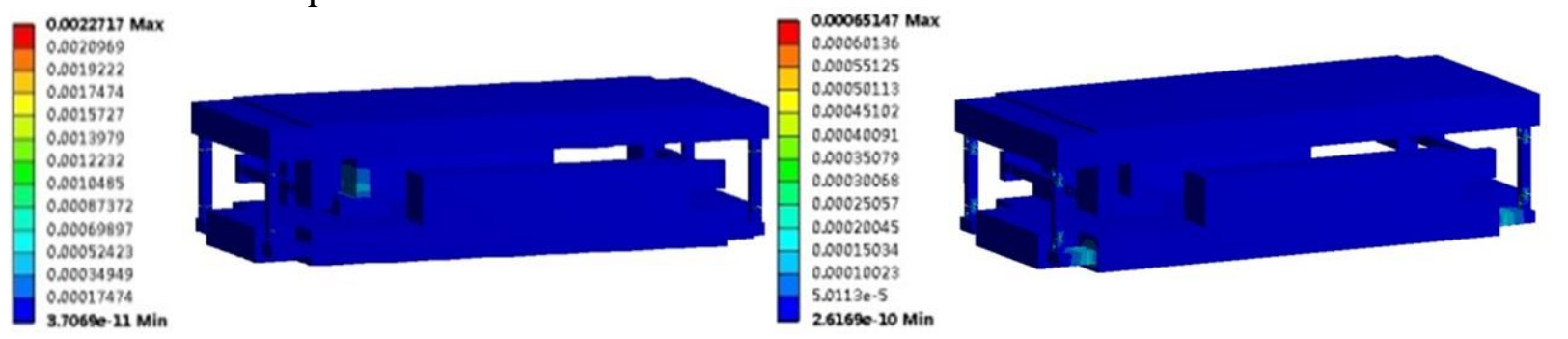

(a) Strain contours of the sensor under the action of Fx action of Fy

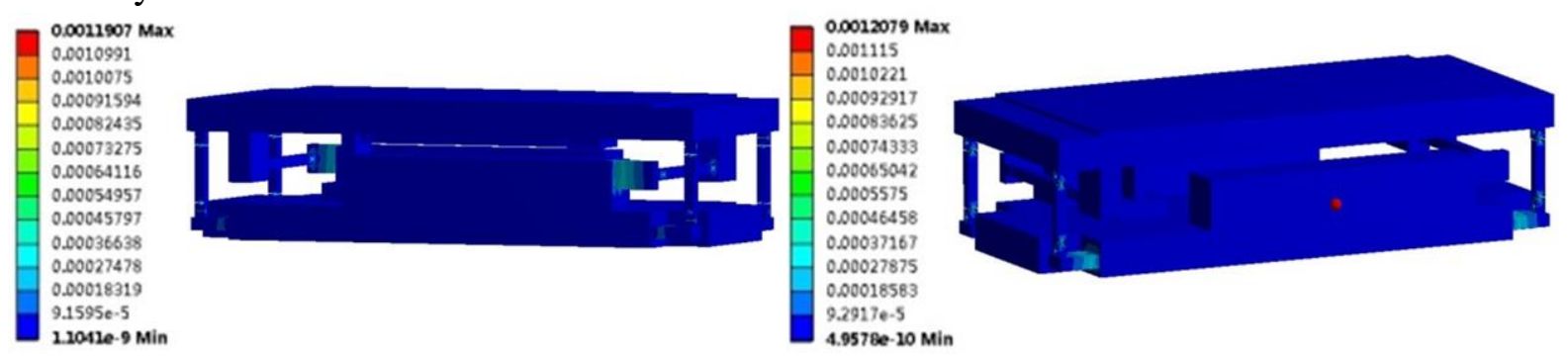

$\begin{array}{ll}\text { (c) Strain contours of the sensor under the action of Fz } & \text { (d) Strain contours of the sensor under the }\end{array}$ action of Mx 


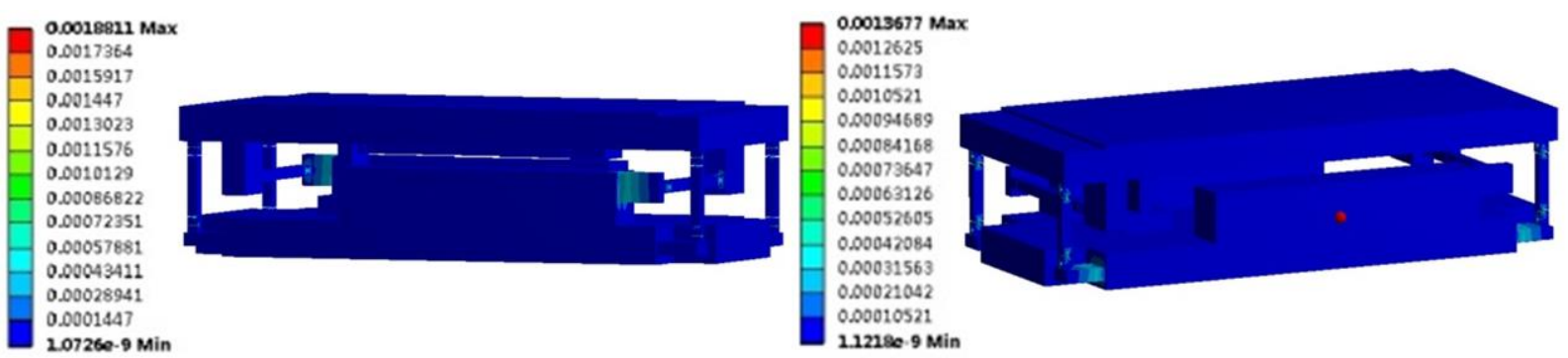

(e) Strain contours of the sensor under the action of My

(f) Strain contours of the sensor under the action of $\mathrm{Mz}$

Fig. 3 Strain contours of the sensor under the action of force and moment in every direction

It is observed that the measuring element is highly sensitive to the load of the corresponding component, and is almost no influence on the load of other components. The unipolarity of the elastic linkage mechanism can effectively block other interference of the strain. The force and moment are relative thoroughly decomposed with the goal of the design.

\subsection{Strength and Deformation Analysis}

The material of the elastomer for the balance is maraging steel $(00 \mathrm{Ni} 18 \mathrm{Co} 8 \mathrm{Mo} 5 \mathrm{TiAl})$, and the yield strength $\sigma_{\mathrm{s}}=1.75 \times 10^{9} \mathrm{~Pa}$. The material of the maraging steel has relatively high strength, fine elastic performance, low coefficient of expansion, high fatigue strength and impact toughness, which can ensure the stability and measurement accuracy for the balance. The stress distribution of the balance is shown in Fig.4. The position of the maximum stress of the balance is located in the dual-arc dual-direction elastic hinge. The maximum stress of the balance is $4.65 \times 10^{8} \mathrm{~Pa}$, and less than the yield strength $1.75 \times 10^{9} \mathrm{~Pa}$.

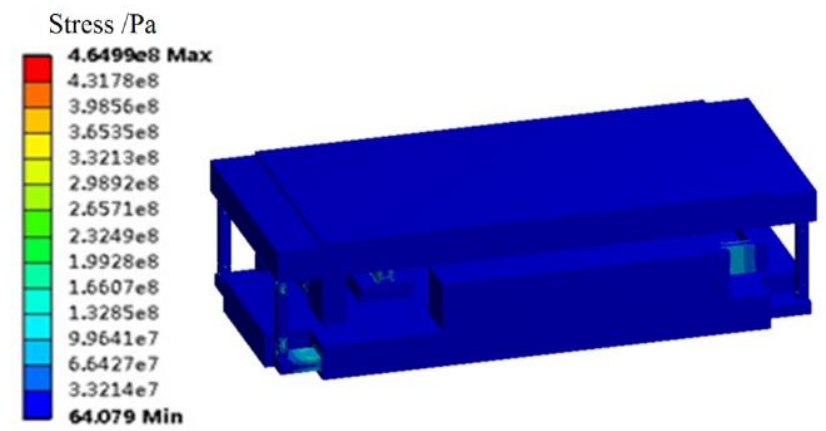

Fig. 4 Stress distribution of the balance

In order to accomplish the force and moment measurement of the balance, it is vital to coordinate the relationship between the stiffness and strain of the balance. The displacement distribution of the balance is shown in Fig.5. It is observed that the maximum linear displacement of the balance is $0.147 \mathrm{~mm}$. The maximum linear displacements are $0.108 \mathrm{~mm}$ in the $x$ direction, $0.0757 \mathrm{~mm}$ in the $y$ direction and $0.071 \mathrm{~mm}$ in the $z$ direction. The maximum angular displacements are $\alpha=1.6^{\prime \prime}$ for the pitching angle, $\beta=0.8^{\prime \prime}$ for the yaw angle and $\gamma=3.6^{\prime \prime}$ for the roll angle. The results show that the values of the results are the same order of magnitudes in all directions and the balance structure is reasonable. It is high stiffness and little deformation without elastic correction.

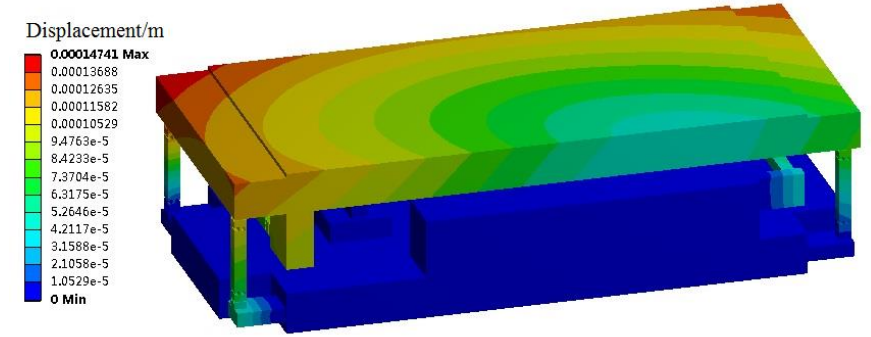

Fig. 5 Displacement distribution of the balance 


\subsection{Modal analysis}

The modal would produce huge vibration when the test starts or stops. If the frequency of the air flow is equal to or close to the natural frequency of the balance system, the balance system would resonate with the experimental model. Therefore, the natural frequency of the balance should be calculated and different from the frequency of the high speed air flow. The bottom of the balance is fixed. That is, the bottom of the balance is bounded with six freedoms in the simulation. The modal analysis of the balance from the first order to the sixth order is shown in Fig.6.

The magnitudes of frequencies are $249 \mathrm{~Hz}$ for first-order in the axial direction, $274 \mathrm{~Hz}$ for second-order in the lateral direction, $310 \mathrm{~Hz}$ for third-order in the yaw direction, $366 \mathrm{~Hz}$ for fourth-order in the normal direction, $678 \mathrm{~Hz}$ for fifth-order in the rolling direction and $777 \mathrm{~Hz}$ for sixth-order in the pitching direction. It is observed that the frequency distribution is relatively scattered, and there is no centralized phenomenon in the frequency distribution, which shows that the balance has the characteristics of fine dynamic structure. The magnitude of first-order frequency is $249 \mathrm{~Hz}$, which meets the test requirements.

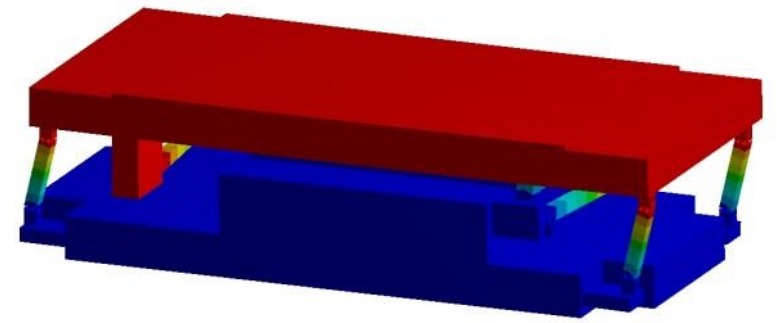

(a) First-order vibration frequency

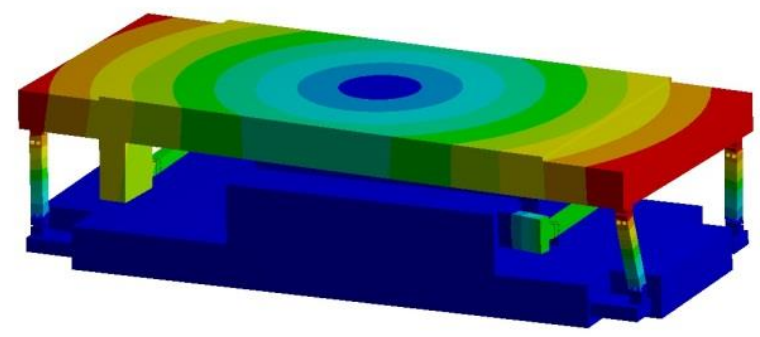

(c) Third-order vibration frequency

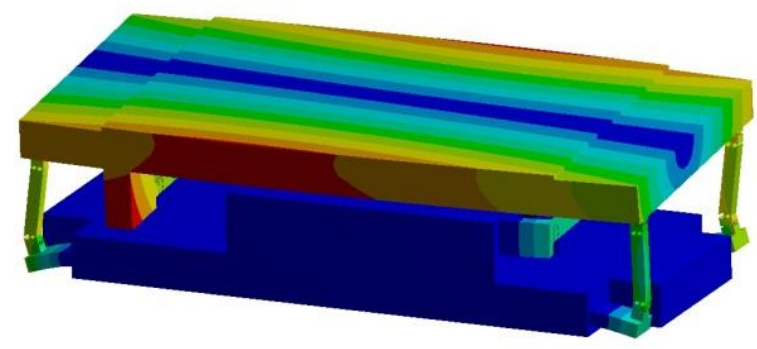

(e) Fifth-order vibration frequency

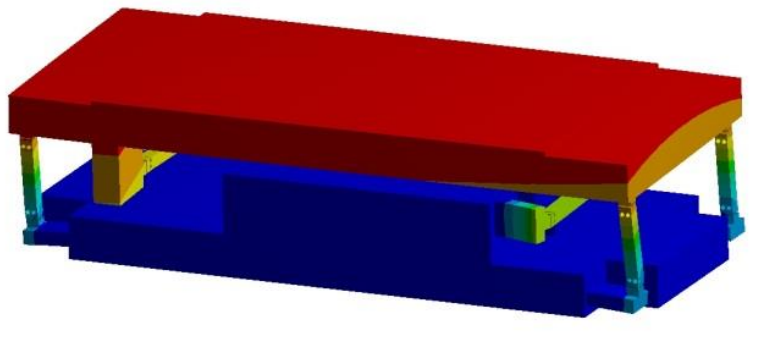

(b) Second-order vibration frequency

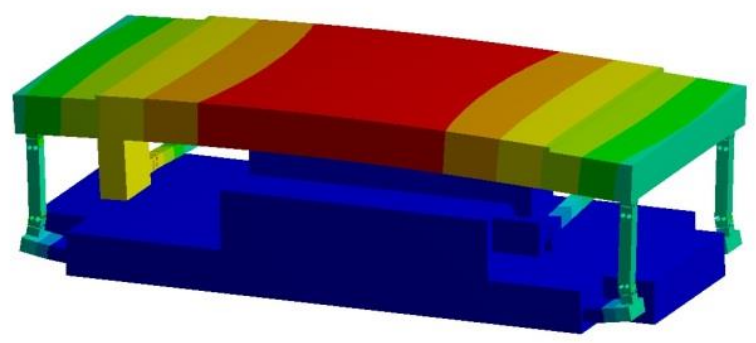

(d) Fourth-order vibration frequency

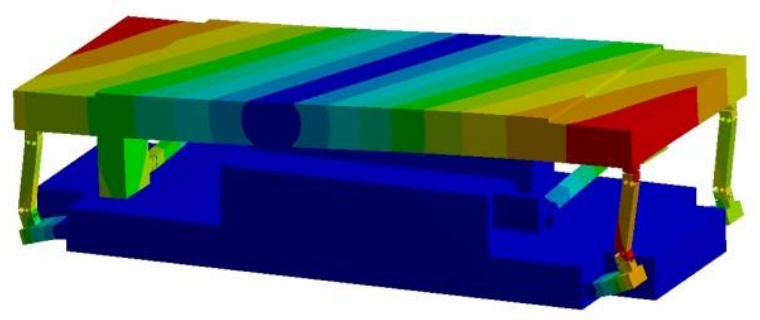

(e) Sixth-order vibration frequency

Fig. 6 Frequency vibration modes.

\section{Balance Calibration and Experiment}

The calibration system of ground axes is adopted for calibrating the balance. The calibration result is listed in Table 1.It is observed that the indicators of the balance are better than those of static calibration index, namely, the indicators of the balance are better than $3 \%$ for precision and 5\% for accuracy. 
Table 1. Static calibration result of the balance (\%oF.S.)

\begin{tabular}{|c|c|c|c|c|c|c|}
\hline & $F_{x}$ & $F_{y}$ & $F_{z}$ & $M_{x}$ & $M_{y}$ & $M_{z}$ \\
\hline Precision & 0.21 & 0.32 & 0.21 & 1.23 & 0.84 & 0.23 \\
\hline Accuracy & 0.42 & 1.25 & 1.13 & 3.1 & 1.12 & 0.43 \\
\hline
\end{tabular}

The comparison of the practical result, the theoretical calculation result and the simulated result are listed in Table 2.It is observed that the theoretical calculation result, the practical result and the simulated result are gradually increscent. There are mainly two reasons leading to such phenomena: (1) the impact of the additional force and moment from the elastic linkage mechanism is neglected in the theoretical calculation result; (2) There is a position error of the strain gauge in the process of the attachment. The calibration and experiment show that the balance meets the requirement for measuring thrust of the mini aero-engine.

Table 2. Signal data contrast of the balance $(\mu \mathrm{V} / \mathrm{V})$

\begin{tabular}{|c|c|c|c|c|c|c|}
\hline & $F_{x}$ & $F_{y}$ & $F_{z}$ & $M_{x}$ & $M_{y}$ & $M_{z}$ \\
\hline Practical results & 798 & 299 & 613 & 582 & 998 & 655 \\
\hline Theoretical results & 841 & 327 & 654 & 595 & 1072 & 676 \\
\hline Simulated results & 804 & 302 & 628 & 576 & 1038 & 664 \\
\hline
\end{tabular}

\section{Conclusion}

1. The box six-component strain balance is firstly adopted to carry out the thrust vector measurement of the mini aero-engine. The layout of balance and the optimization design of measuring element parameters are presented considering the sensitivity and stiffness of the balance for the mini aero-engine.

2. The calibration and experiment results of the balance shows that the measuring error of the balance for each component is lower than $0.3 \%$, and the error of the measuring accuracy is lower than $0.5 \%$. The simulated and experimental results demonstrate that the six-component strain balance meets the requirements of thrust vector measurement for the mini aero-engine.

\section{References}

[1] W. Chen, F.R. Du, S.T. Ding and Y.Q. Li "General-structural design study of a micro turbojet engin,” J. Aerosp. Power, vol. 25, pp. 169-174, 2010. (in Chinese)

[2] T. Zheng, "An introduction to development of turbojet and turbofan engines for missiles," J. Propul. Technol., vol. 16, pp. 7-12, 1995. (in Chinese)

[3] B.Y. Sun, M. Qian and J. Zhang, "Review and prospect on research for piezoelectric sensors and dynamometers," J. Dalian Univ. Technol., vol. 3, pp. 127-133, 2002. (in Chinese)

[4] Z.Y. Wang, B.Y. Sun, J. Zhang, M. Qian Y. Zhai and Z.J. Ren, "Calibration on rocket thrust vector measuring system," Transducer Microsyst. Technol., vol. 26, pp. 64-66, 2007. (in Chinese)

[5] H.Y. Luo, M.J. Hou, Y.G. Wang, W. Ye and Z.G. Liu, "Experime-nu and researches in six-component balance for metal bellows expansion joints," J. Aerosp. Power, vol. 28, pp. 736-742, 2013. (in Chinese)

[6] C.A. Yu, L. Wang, X.Z. He, R.K. Qiu and C.W. Yao, "Design on structure of six-component box balance for the aero-engine vector nozzle measuring platform," J. Aerosp. Power, vol. 31, pp. 23-30, 2016. (in Chinese) 